\title{
Design and Development of Virtual Dressing Room System Based on Kinect
}

\author{
Ka Wai Mok, Chon Teng Wong, Sio Keong Choi and Li Ming Zhang \\ Faculty of Science and Technology, University of Macau, Macau 853, China \\ E-mail: mb65480@umac.mo,wxo_dennis98z@hotmail.com, fairy_0068715@hotmail.com,lmzhang@umac.mo
}

Received: 20 June 2017; Accepted: 06 August 2018; Published: 08 September 2018

\begin{abstract}
This paper proposes a Microsoft Kinect-based real-time 3D virtual dressing room system. The system has two advantages. First, by using Kinect and 3D modeling customization, the user's real-time movement is tracked and displayed, which provides the user a realistic feeling during trying on the items virtually. Second, unlike other virtual dressing room where the user has to use it physically at the boutiques, the system can be used at home, which provides the users more convenient shopping experience. Our main goal is focusing on the garment physical movement, by different techniques, we try to make the physical movement of the garment as real as we can, because we think that the physical movement of the garment is one of the most important standard to classify a good virtual dressing system. The paper is divide into seven parts, include introduction, important techniques, system description, result and conclusion etc., also we included some related work that we have searched to improve our system.
\end{abstract}

Index Terms-Virtual dressing room, Kinect, 3D model customization, garments design, motion detection.

\section{INTRODUCTION}

There are many virtual dressing room systems and studies nowadays and most of them are focusing on the hardware mirrors in the store and a lot of money is spent to build up different mirrors. Zugara [1] and wearIT [2] are virtual dressing room companies which provide virtual dressing systems on different platforms. Dulaji Hidellaarachchi et al. [3] suggested an ICT based solution for virtual garment fitting for online market place Yet there is one drawback - they just project the image of the garments onto the customer, and most of the time the original outfit of the customer will affect the outcome of virtual dressing, hence the customer's realistic feeling decreases. To solve this problem, we propose a virtual dressing room design by using Kinect and 3D modeling customizations. Our idea focuses on recording the live movement of the user by using Kinect and let the user experience a realistic feeling of trying on the clothes. Meanwhile, Kinect is not an expensive tool and our approach is to get a high-quality virtual dressing room at home with fewer budgets.

\section{RELATED WORKS}

As we have mentioned in section I, there are many virtual dressing room systems, so when we are doing the research. We focusing a lot into the technique that can improve our system, here are some of the related information and techniques:

\section{A. Kinect}

Kinect was launched in 2010 and has then become the main depth image sensor in many applications. Its function is to track the movement of the user. Kapil S. Raviya et al. [4] shows the arrangement of a Kinect sensor, consisting of an infrared (IR) projector, an IR camera, and a color camera. The depth sensor comprises the IR projector and the IR camera. The IR projector casts an IR speckle dot pattern into the 3-D scene while the IR camera captures the reflected IR speckles. Maccormick [5] introduces the basic structure of Kinect, such as different cameras and their function. Givonni et al. [6] compare the two skeletal tracking SDKs, OpeNI and Kinect for Windows SDK. Philipp Presle [7] brings in the idea of using depth camera to build a virtual dressing room, but the quality of recording live movement was not so good which might affect the performance of the virtual garments as well as the realistic feeling of the try-on experience. Zhou et al. [8] propose a virtual dressing room using Kinect, where Kinect is used to track the user's skeleton, then the clothes image or 3D model is projected onto the body of the user.

\section{B. Garment design}

For a virtual dressing room, garment design is the most important technique. Gahan's Autodesk 3ds MAX tutorial [9] introduces a solution. One can use Garment Maker to create paper patterns in the panel, join the patterns by seams, and then simulate the paper patterns until they fit on the body. However, this method is quite difficult and time-consuming in the matching process. On the other hand, while using Marvellous Designer [10], cloth and garments are created quickly with good results. One just needs to draw the paper patterns of a garment, join them with seams, simulate it and then the garment will match the body automatically. Therefore, we decide to use the later software for our garment design. 


\section{Facial capture}

Another important feature of our approach is the facial capture of the user. As our system is designed as a membership system, every user will has his or her avatar in the virtual dressing room. Hence, we need to create a unique $3 \mathrm{D}$ model for every user and it is necessary to have a facial capture of him or her. For this, we have considered two software tools, Looxis Faceworx [11] and Autodesk 123D Catch [12].

Looxis Faceworx [11] is an application which can help to generate the 3D figure of a face from two photographs taken from two different angles, and to be specific only one front image and one side image are needed. After the photographs are taken, the next step is to match all the key points of the face in the two photographs, such as eyes, ears, mouth, nose, forehead, cheeks, and so on. A virtual face object will then be generated. Looxis Faceworx is very easy to use, yet the quality is not so high.

On the other hand, Autodesk 123D Catch [12] is a program which creates 3D models from a series of photographs taken from various angles using photogrammetry. In other words, it creates a $3 \mathrm{D}$ model out of multiple pictures of the user. Catch can be used to create 3D models for people, places, and objects as well. By using Autodesk 123D, we can create a super-realistic virtual facial model for the user, and this will be discussed in the later section.

\section{IMPORTANT TECNIQUES}

Aside from using Kinect camera, we have searched for a lot of methods and techniques to create the user model to improve the performance of our system. In this part, we will talk about some related parts about Kinect camera, creation of the user model, especially the user face and hair capture, as well as cloth simulation.

\section{A. Set up of a Kinect camera}

After the creation of the 3D model, we need to set up our camera - Kinect. According to the guideline of Microsoft in 'Setting up a Kinect Sensor' [13], first we need to place the Kinect on a stable surface and download Kinect for Windows SDK, then plug in the Kinect sensor. After connecting to the Kinect sensor we can get three types of streams, namely color, depth and skeleton stream. Our goal is to let the user select the clothes he/she wants after logging in, and then the model and the clothes will be shown on the screen as long as he/she stands in front of Kinect. The user can then see his/her virtual image wearing the clothes he/she has chosen. The experiment results show that our system is very practical with acceptable quality.

\section{B. Face and hairstyle capture}

To increase the realistic feeling of the try-on experience for the user, we need to capture the details of the user's head, including face and hairstyle. To build a good model of the user's avatar, we have to use photos to construct a 3D model object. The following are the methods to build up a 3D model object from photos.

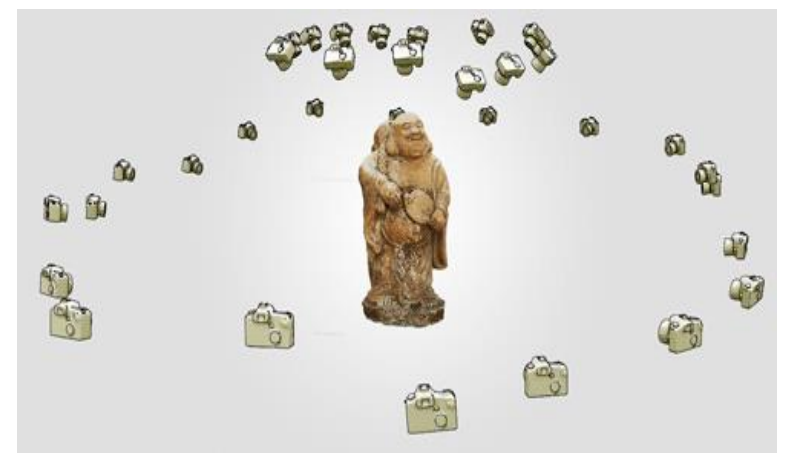

Fig.1. The main workflow of Autodesk 123d Catch, capture from [12]

As mentioned above, Autodesk 123d Catch [12] is a program which creates 3D models from a series of photographs taken at various angles using photogrammetry. This program uses photogrammetric technology to create a 3D model out of multiple pictures of the user. Before creating a 3D model in Autodesk 123d Catch, the user should find a place that allows him/her to be photographed from many angles under good light source. It is recommended to take around 30 to 40 pictures in order to create a well-rounded model.

It is harder to capture a real face and head model than to capture a stationary object. As people may not be able to stay perfectly still during the photo taking, people might move a little bit. And this is the most problematic issue in this process since any movements during the photo taking will influence the quality of the model.

In the example of the below Fig. 3, it shows that one of the edit tools is called smooth tool. We can use this tool, Autodesk Meshmixer [15] to smooth out the details on the selected area of the mesh. So that the main use of Autodesk Meshmixer is for tidy up a 3D model, for example, to remove areas that are not wanted or to fill holes. It can also be used to sculpt the shape or correct the mistaken part.
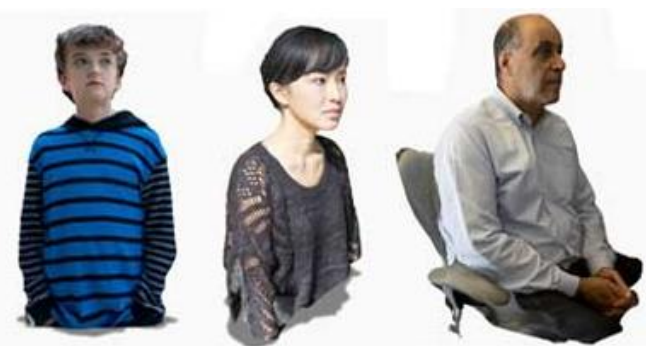

Fig.2. To capture the user head, the user should sit still for a while, capture from [12]
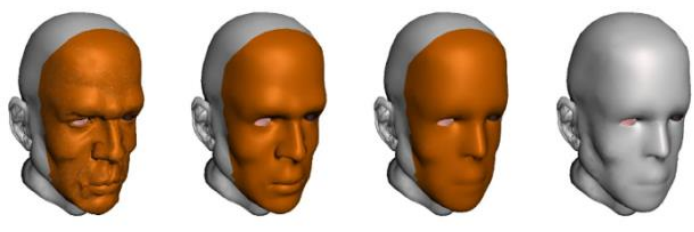

Fig.3. To smooth the face detail of a head $3 \mathrm{~d}$ model 
Also, Autodesk Meshmixer [15] is a design tool that allows a lot of customization and modification. It can import different objects and mix them together.

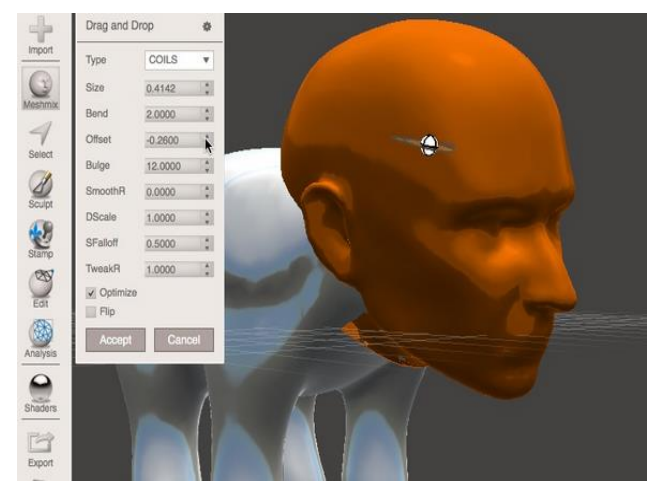

Fig.4. Mix a 3d head model into others $3 \mathrm{~d}$ model

\section{Cloth simulation}

This is the most important part of our project. To increase the realistic feeling of the user when they are using our machine, the performance and presentation of the cloth under different physical movement of the user are very important.

In different software engines, they have different cloth simulation engines and processes, for example: Unity3D [14], which is a game development platform in widely use. Its cloth simulation has a lot of values that can allow the designer adjust like Fig. 5 and 6 . The setting part will be explained in the later section.

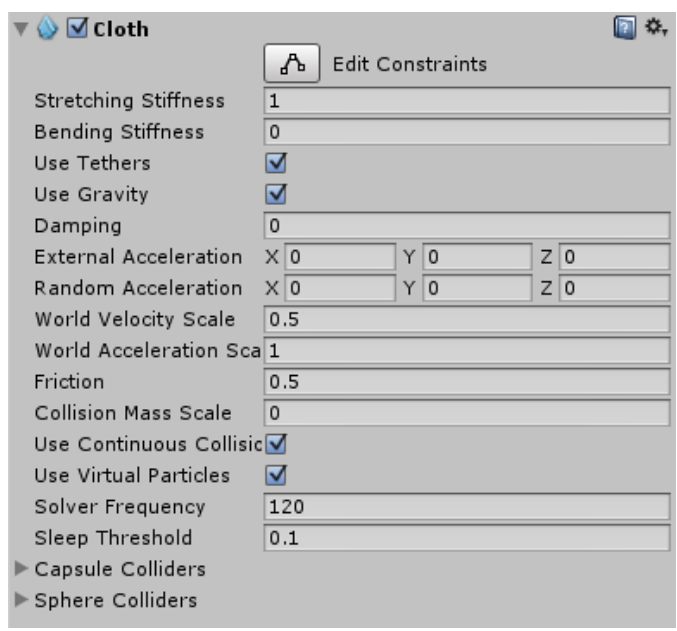

Fig.5. Cloth element in Unity3D

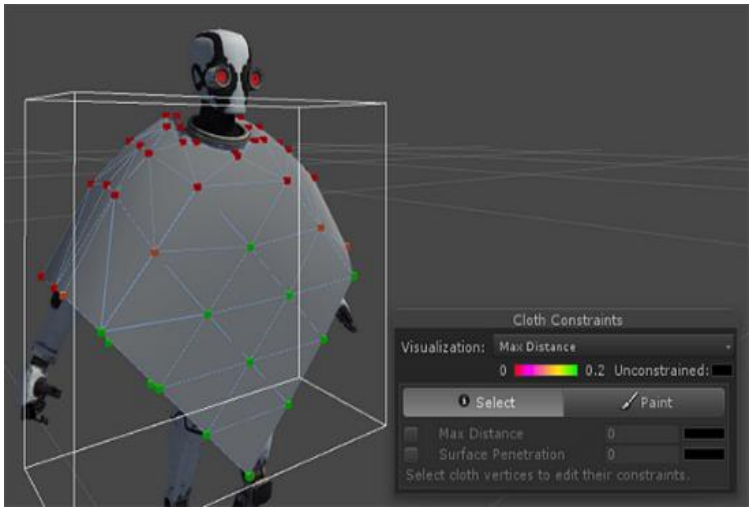

Fig.6. Setting cloth constraints in Unity3D

After all the research of the related work and survey, we found that there are many similar projects and researches in different countries. Some talk about the realistic effect of the cloth while some focus on the movement of the avatar. After all we start to implement the system by ourselves. In the following sections, we will show how we implement the virtual dressing room and we will use ourselves to be the simulated users.

\section{PRINCIPLE OF THE PROPOSED KINECT VIRTUAL DRESSING ROOM SYSTEM}

In this section, we will talk about the basic information about our system, such as the workflow, the way to use our system, the advantages we have and so on.

Our proposed system can be installed in the boutiques or even at homes if the user has a Kinect at home. The main flow chart of our system is as follows.

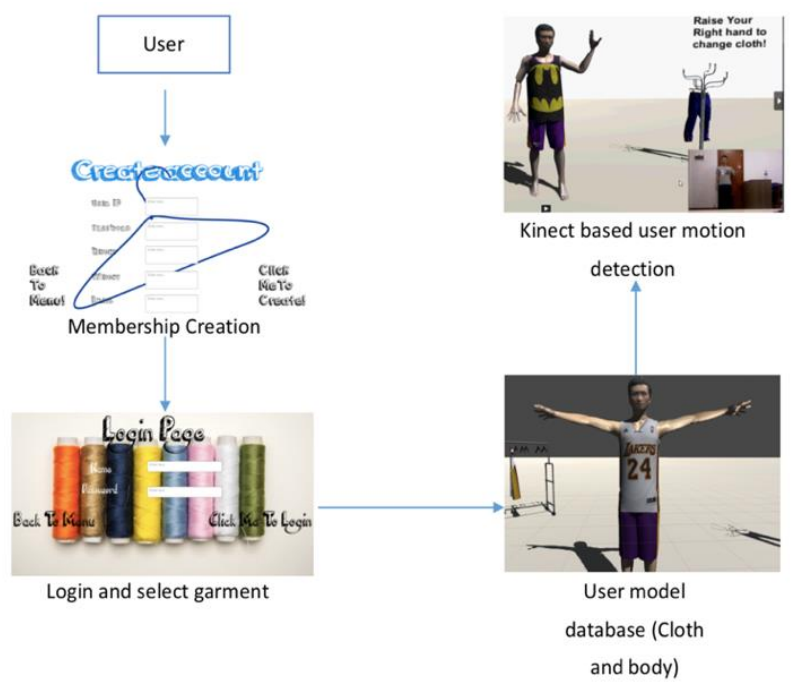

Fig.7. The main workflow of the system 
In our system, we have created a GUI for the user to interact with our virtual dressing room. The user can select garments via the interface, and behind the interface, we have garment database and user body database.

When a user wants to use our system, he/she first needs to register with our system for a membership application. The user needs to apply for the membership at the boutiques. The staff will help to do the following jobs.

1) Capture the facial detail of the user

2) Measure his/her body size and record the basic information of the user in order to create a realistic model of the user.

After the membership is created, the user can start to login the system at home. The user can control his/her own 3D model, and Kinect is used to record the data in real time.

\section{SYSTEM DESCRIPTION}

In this section, we will explain the main and important parts of our system, such as membership, the user model, and the connection between our model and the Kinect motion detection data. These parts are the most important in our system, because the main different between our system with other virtual dressing is the realistic feeling of the user. The main part to improve is the quality of the user model so in the next part we will explain what skills we used to create our models and system.

\section{A. Membership Creation}

Fig. 8 shows that in order to use our system, every user needs to register with our system. We will collect some basic data of the user's body size, such as height, weight, arm span and other basic measurements. We will capture the head and hair style of the user as well, for generating a realistic 3D model of the user.

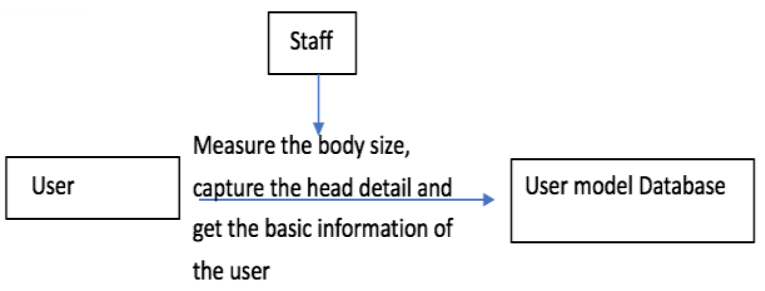

Fig.8. The workflow of membership creation

\section{B. Login and Select Garments}

Fig. 9 shows the main GUI of our system. The user can login the system and select some garments that he/she wants easily through the interface.

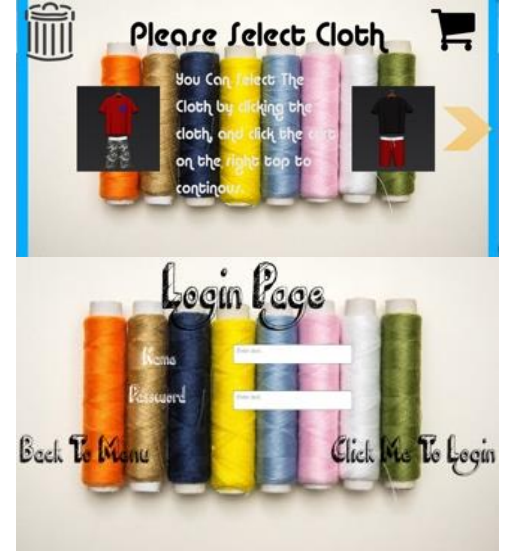

Fig.9 The interface of the system for login and garments selection.

\section{User Model}

In order to create a good user model, there are two main part, the user body model (head and body $3 \mathrm{~d}$ model), and garment design and creation. So in this part we will explain the steps to create $3 \mathrm{~d}$ user model.

\section{User body model design}

First, we need to capture the details of the user's head to increase the realistic sense. To do that, we use Autodesk 123D Catch [12] to assist us in transforming 2D photos into 3D models. We need a series of photos of the head as shown in Fig. 10 and the generated 3D head model is shown in Fig. 11

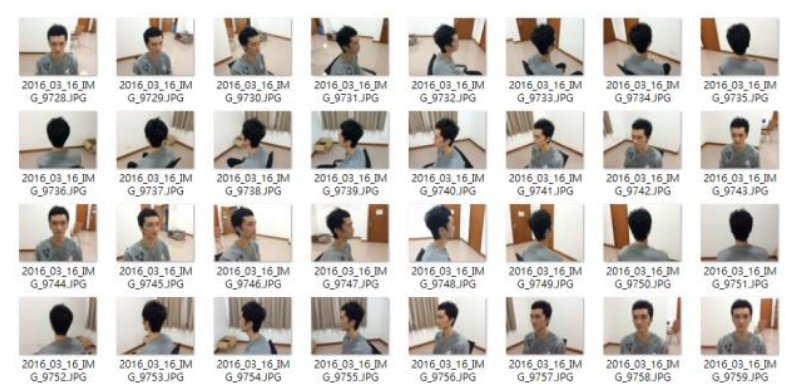

Fig.10. A series of photographs of our group member

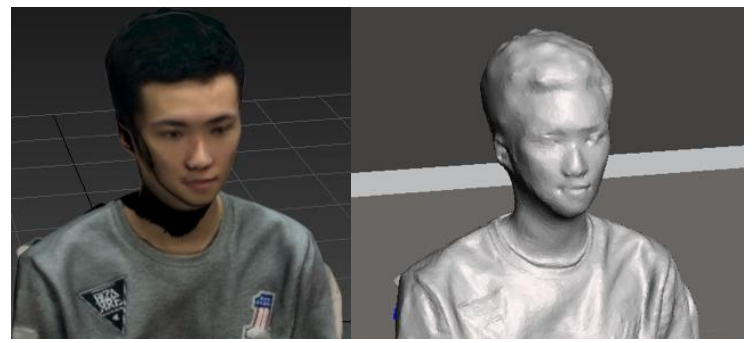

Fig.11. Generate the 3D head model 


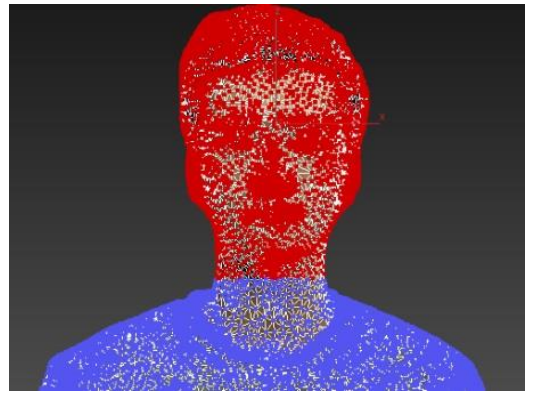

Fig.12. The selected part of the head

Next part is to detach the head part which we only need. For 3D modelling editing, we use 3ds Max [17] (user-friendly and professional), in which we can modify the mesh by selecting the vertices, side, area, etc. This time we are going to use vertices because they can cover more area and make the outcome more meticulous. And after we select a specific part, we can delete other parts which are not needed.

After all, we get the specific part of the model, and that indicates we have completed the most important part of the avatar.

At last, we are going to combine the head and the body together. To produce the body, we just need to measure the basic body measurements of the user, and then input the body parameters into some software (i.e. Marvellous Designer, Fuse) and then the software can give us a very real body and the measurement is done in the user register stage. Since the body part is not our focus, we will not discuss it in detail in this paper. After we get the body, we need to attach the head to the body. We have to adjust the size of these two objects and let them match together as shown in Fig. 14.

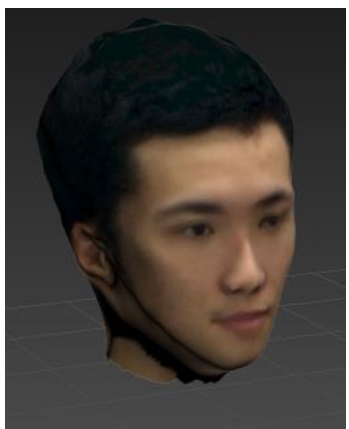

Fig.13. A specific head model

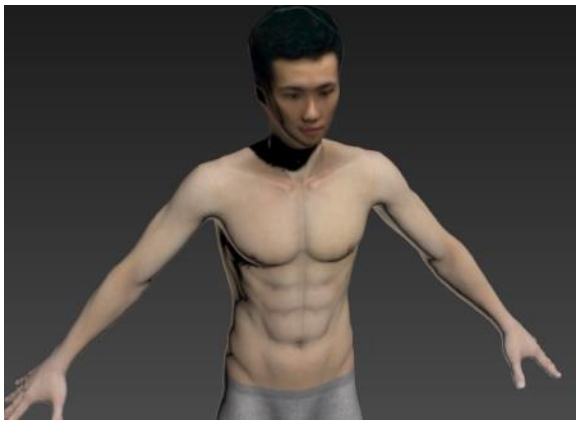

Fig.14. A whole customized user body model

\section{Garments model design}

For garment creation, there are so many software which can help us to do that. In our system, we choose Marvellous Designer [10]. First we need to create the clothes patterns, like Fig. 15. On the patterns, we can add a lot of images, figure or words.

After we create the pattern, we need to connect them together so we need to create the seam. it is use to connect two edges of the clothes by a set of lines part by part. It is important that which part should connect to which part.

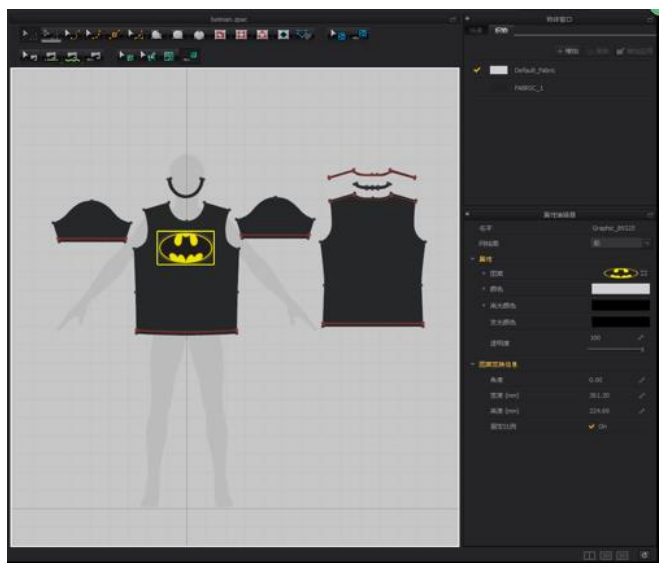

Fig.15. Customization of a T-shirt

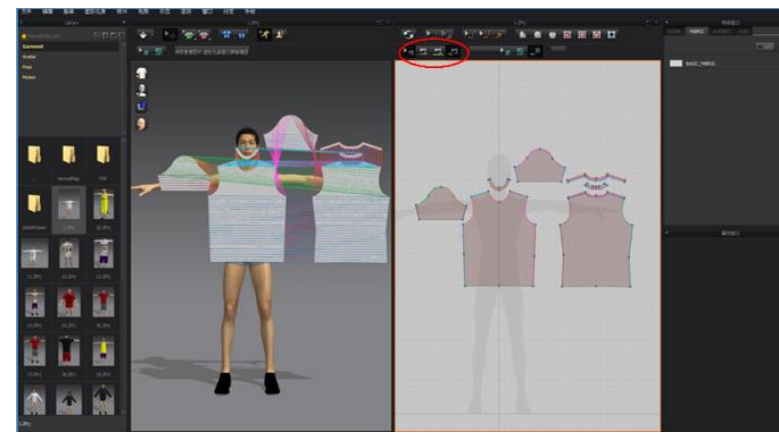

Fig.16. Create the connection of the T-shirt

After the connection of the pattern, we can create a complete garment and output the model.

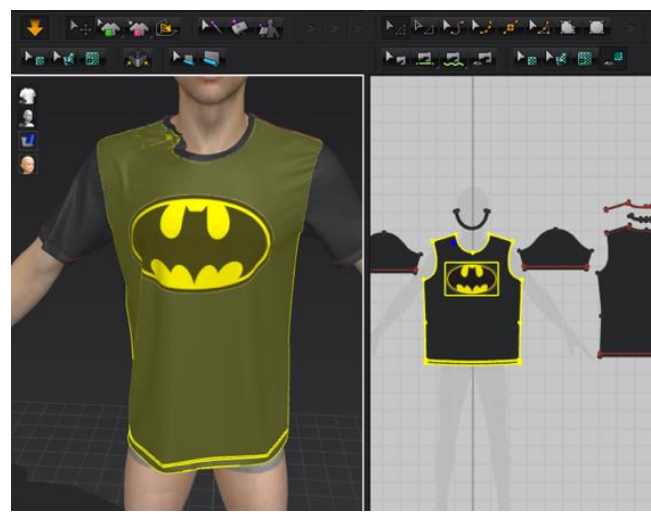

Fig.17. A full T-shirt. 


\section{Cloth Simulation}

Cloth simulation is very an important part in our system. A good cloth simulation can perform a very realistic physical performance of the clothes. We use Unity3D [14] to be our platform to perform the simulation.

First of all, import the model into the scene in unity $3 \mathrm{~d}$. Unity3d manage game in a scene view, from one scene to another, just like we playing game, from one stage to another. Because cloth is one component of the model, so when we can add some unity $3 \mathrm{~d}$ element of the shirt, this time 'Cloth' element.

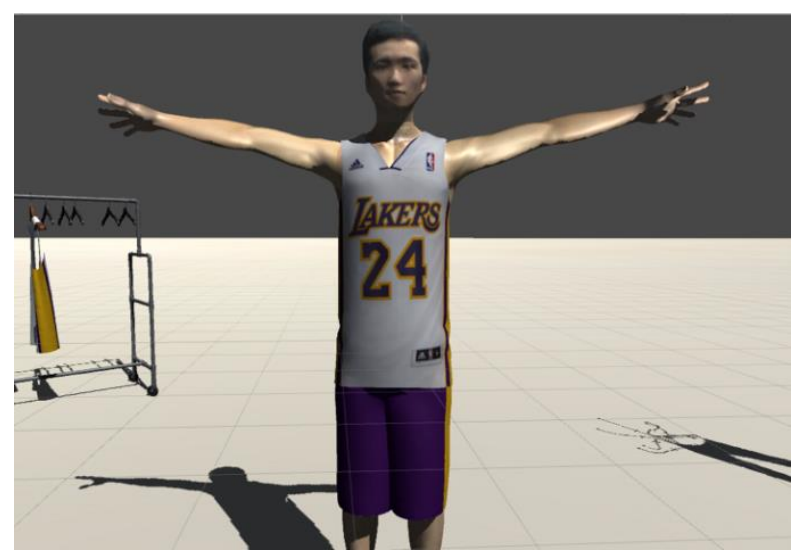

Fig.18. A Full user model import into Unity3D scene

Most of the parameters is about how tight or lose of the cloth, the main parameters we focus on is the cloth constraints and the colliders of cloth. All other parameters we keep it same as the default. Cloth constraints is to set which vertex of the cloth need to be fix and which vertex need to be un-fix. Let's take a look at the image to for further explanation.

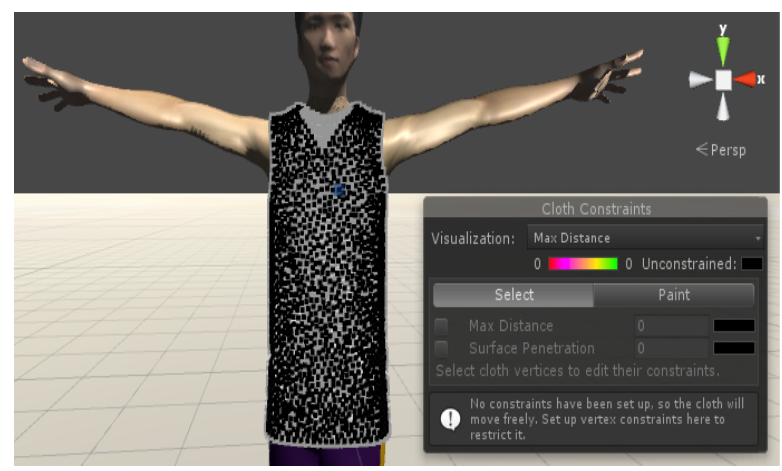

Fig.19. Setting the cloth constraints I

In Fig. 19, we can see that because the constraints don't set, so the vertex of the cloth is in black color. So we need to set the cloth constraints, first of all we set the vertex of the top of the shirt be 0 , that is

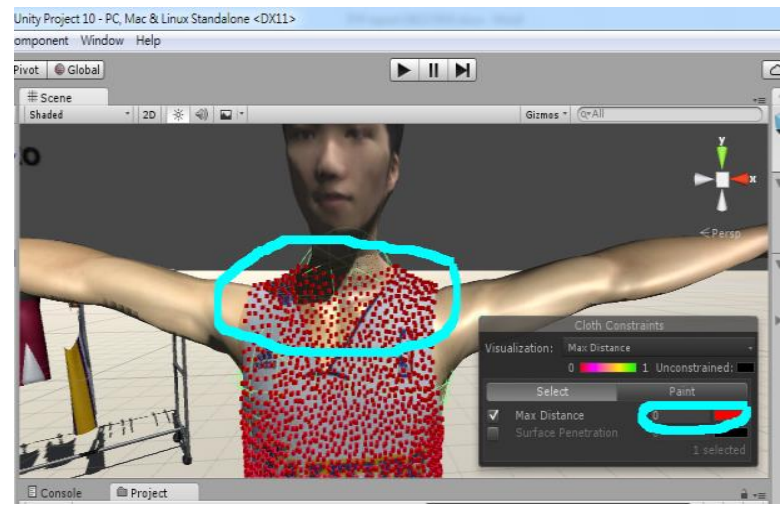

Fig.20. Setting the cloth constraints II

After that set other vertex value to 0.05 ,

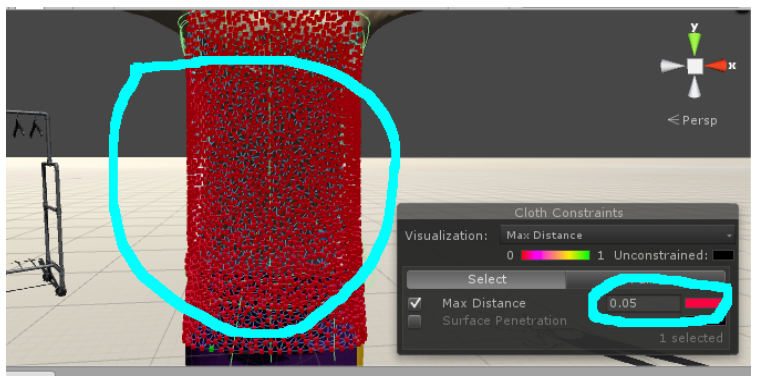

Fig.21. Setting the cloth constraints III

This setting is the most suitable setting that we can find to fit in our system, after the cloth simulation, we can connect the full user model with cloth simulation with the Kinect based motion data.

\section{E. Kinect Based Motion Detection}

To connect the live movement collected by Kinect with the user model, we need to construct a skeleton for the user model. Fig. 22 shows an example of the user body with clothes which match the size of the body after we put them together (A full user model).

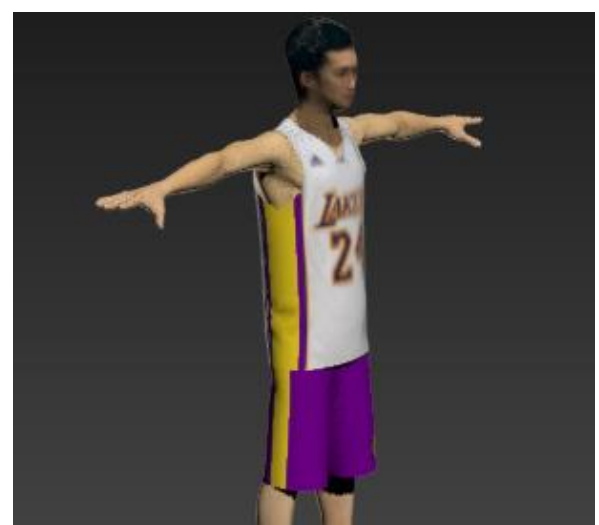

Fig.22. Example of a basketball jersey 
To construct a skeleton for the user model, we use a professional platform Mixamo [16], which can build a very good skeleton for the user model. In Mixamo [16], we can upload any humanoid bipedal characters to the platform. After we select several basic skeleton points, Mixamo [16] will automatically generate the whole well structure skeleton of the model. In Fig. 23 we can see that Mixamo [16] created a very accurate skeletal model, after that we can start to connect the full user model with the Kinect detection data.

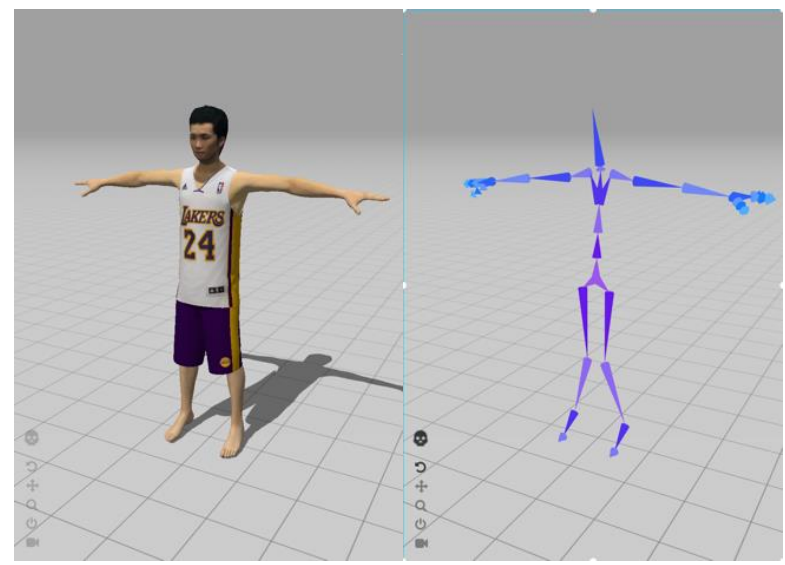

Fig.23. A skeleton built by Mixamo

To connect with Kinect and display the final result, we choose the platform Unity3D [14]. Unity3D is a well-known game development platform, which can develop 2D and 3D games, and due to the advanced technology, Unity3D can also implement VR game lately. In order to achieve a realistic result of the model, we choose Unity3D as our display platform. To connect with Kinect, we need some basic settings for our model and some programming to capture the gesture of the user and perform certain actions as shown in Fig. 24.

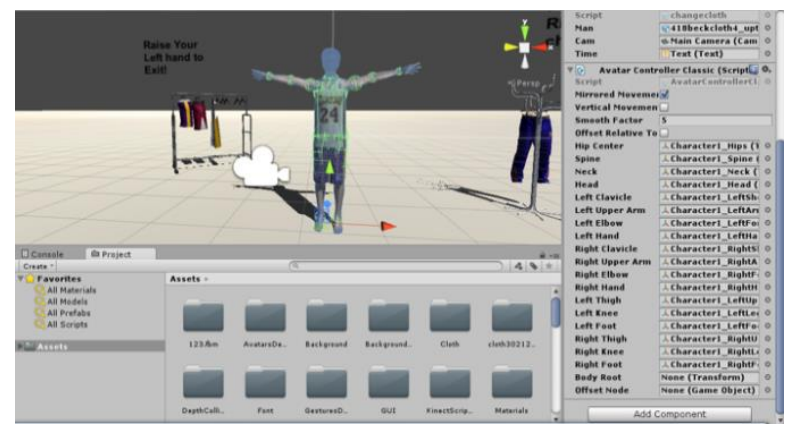

Fig.24. Connecting a user model with the setting of Unity3D

\section{RESULTS}

After the explanation of our system, here we will show the result of our system. In Fig. 25, we show different clothes and different user models. After the connection between Kinect and our model, the user can move in front of the Kinect camera and control the user model in the system, with some very realistic clothes movements.

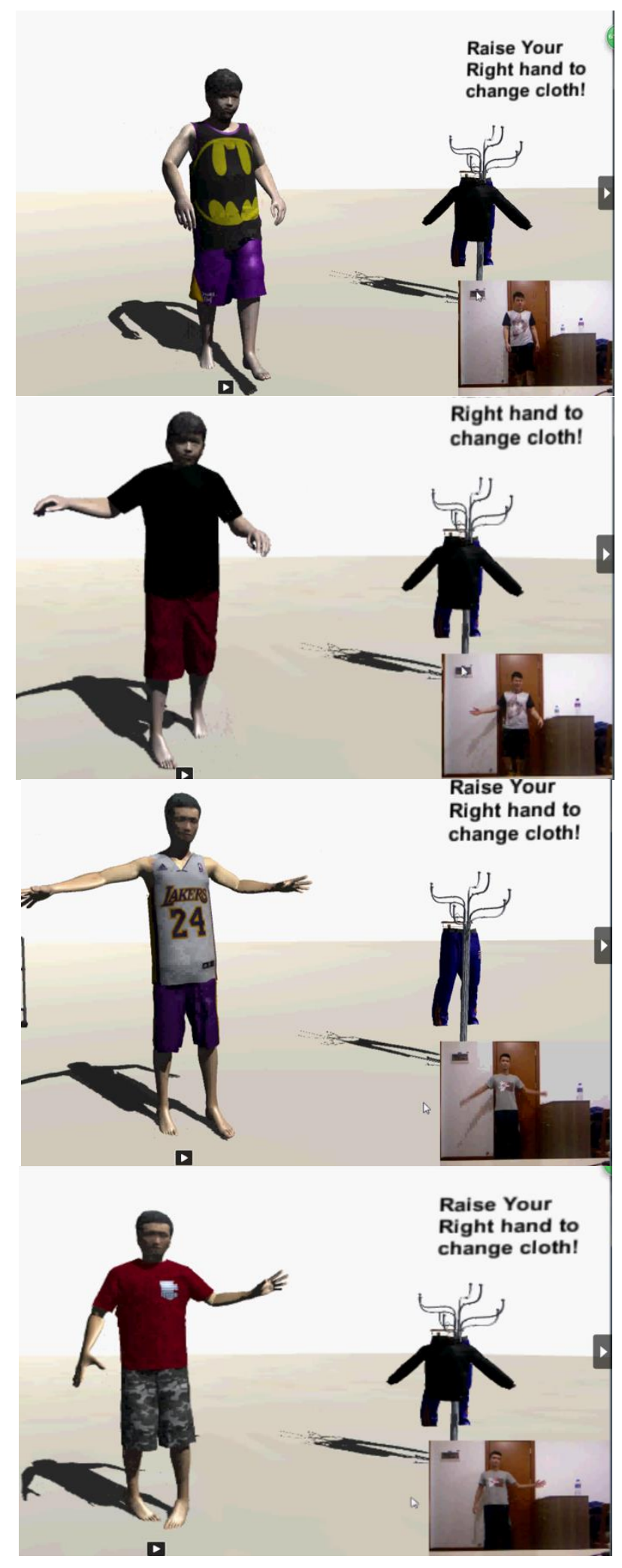

Fig.25. Result of our system

\section{CONCLUSION}

In this study, we designed a virtual dressing room with low cost and high convenience for the customers using at home. The system contains several models with very 
realistic appearance, movements, and garments. Also, we have designed a very user-friendly interface. With some basic input and Kinect, the users can try on any garments that he/she likes in our virtual dressing room conveniently.

\section{REFERENCES}

[1] Zugara. [Online], Retrieved from: http://zugara.com/virtual-dressing-room-technology, access time: $2018 / 7 / 5$

[2] wearIT. [Online], Retrieved from: http://coitor.com/wearit-virtual-dressing-room/, access time: $2018 / 7 / 5$

[3] Hashini Gunatilake, Dulaji Hidellaarachchi, Sandra Perera, Damitha Sandaruwan, Maheshya Weerasinghe, "An ICT Based Solution for Virtual Garment Fitting for Online Market Place", International Journal of Information Technology and Computer Science(IJITCS), Vol.10, No.2 pp.60-72, 2018. DOI: $10.5815 /$ ijitcs.2018.02.06

[4] Kapil S. Raviya, Dwivedi Ved Vyas, Ashish M. Kothari,"Real Time Depth Data Refurbishment in Frequency Domain and 3D Modeling Map Using Microsoft Kinect Sensor", International Journal of Image, Graphics and Signal Processing(IJIGSP), Vol.8, No.11, pp.49-58, 2016.DOI: 10.5815/ijigsp.2016.11.07

[5] John MacCormick, "How does the Kinect work?",Talk pdf of John MacCormick, Dickinson College, 2011

[6] Giovanni, S., Choi, Y. C., Huang, J., Khoo, E. T., and Yin, K. 2012. "Virtual Try-on Using Kinect and HD Camera." In Proceedings of MIG 2012, 55-65

[7] Philipp Presle, "A Virtual Dressing Room based on Depth Data", Master thesis of faculty of informatics, Vienna University of Technology, 2012

[8] Zhou, Z., Shu, B., Zhuo, S., Deng, X., Tan, P., and Lin, D.2012. "Image-based Clothes Animation for Virtual Fitting." Presented at the SIGGRAPH Asia 2012 Technical Briefs, Singapore.

[9] 3Ds Max Modeling for Games: Insider's Guide to Game Character, 2011, Andrew Gahan

[10] Marvelous designer. [Online], Retrieved from: http://www.marvelousdesigner.com, access time: 2018/7/5

[11] Looxis Faceworx, 2007, [Online], Retrieved from: http://looxis-faceworx.en.uptodown.com, access time: 2018/7/5

[12] Learn how to use 123d Catch, [Online], Retrieved from: http://www.123dapp.com/howto/catch, access time: 2017/6/14

[13] Setting Up a Kinect Sensor, [Online], Retrieved from: https://msdn.microsoft.com/en-us/library/hh855356.aspx, access time: 2017/1/22

[14] Unity3D, 2017, [online], Retrieved from: https://unity3d.com, access time: 2018/7/5

[15] Autodesk Meshmixer, [Online], Retrieved from: http://www.meshmixer.com, access time: 2018/7/5

[16] AUTO-RIGGER (PREVIEW), [online], Retrieved from: https://www.mixamo.com/, access time: 2018/7/5

[17] Draw the Patterns for the Clothes, 2014, [Online]. Retrieved from: https://knowledge.autodesk.com/support/3ds-max/gettingstarted/caas/CloudHelp/cloudhelp/2015/ENU/3DSMax-Tu torial/files/GUID-02B657B3-74DC-44E1-89D7-AEF8C1 9BDEBB-htm.html, access time: 2018/7/5

\section{Authors' Profiles}

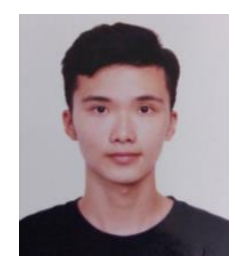

Ka Wai, Mok obtained his BSc. degree in Computer Science from Faculty of Science and Technology of University of Macau in 2016.

$\mathrm{He}$ is currently studying MSc. Degree in Computer Science at Faculty of Science and Technology of University of Macau. His research interests are image processing, 3D modeling, computer vision and depth extraction.

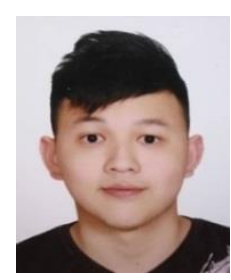

Chon Teng, Wong obtained his BSc. degree in Computer Science from Faculty of Science and Technology of University of Macau in 2016.

$\mathrm{He}$ is currently work in Star World Macau, as a surveillance.

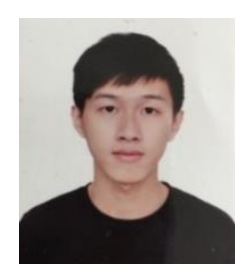

Sio Keong, Choi obtained his BSc. degree in Computer Science from Faculty of Science and Technology of University of Macau in 2016.

$\mathrm{He}$ is currently work in Tai Fung bank in Macau, as an auditor.

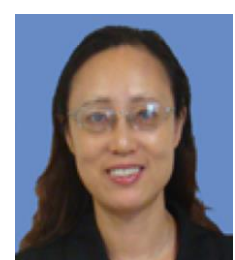

Li Ming. Zhang, received the B.S degree in Computer Software at Nankai University, China and M.S. degree in Signal Processing at Nanjing University of Science and Technology, China. She received her Ph.D. degree in image processing at University of New England, Australia.

She is currently an assistant professor in Faculty of Science and Technology, University of Macau. Her research interests include signal processing, Image processing, Computer vision and multimedia computing.

How to cite this paper: Ka Wai Mok, Chon Teng Wong, Sio Keong Choi, Li Ming Zhang, "Design and Development of Virtual Dressing Room System Based on Kinect", International Journal of Information Technology and Computer Science(IJITCS), Vol.10, No.9, pp.39-46, 2018. DOI: 10.5815/ijitcs.2018.09.05 